\title{
MODIFIED LINEAR-QUADRATIC REGULATOR USED FOR CONTROLLING ANTI-TANK GUIDED MISSILE IN VERTICAL PLANE
}

\author{
ŁukAsz Nocoń, ZBigniew Koruba \\ Kielce University of Technology, Kielce, Poland \\ e-mail:lnocon@tu.kielce.pl; ksmzko@tu.kielce
}

\begin{abstract}
The paper concerns the issue of optimum control of the strongly non-linear dynamic system, i.e. Anti-Tank Guided Missile (ATGM). The linear-quadratic regulator (LQR) was used to provide control capabilities. In order to use the classic LQR, the dynamics of the object must be presented in the form of a linear-stationary model. This is not possible in the case of the considered missile, mostly due to mass changing in time (intensive consumption of fuel) and varying aerodynamic conditions depending on the Mach number Ma. Thus, we are dealing with a non-stationary system. Moreover, state variables are frequently involved in complex functions, which do not allow one to separate coefficients related to state variables very easily. In order to linearize such a complex system, the paper uses Jacobian, as the matrix of state, calculated at each time instant. The automatic pilot of the ATGM, using the LQR method, determines the signals controlling the angles of flight control surfaces and the thrust vector using continuously calculated Jacobians. The paper presents the algorithm for the ATGM control.
\end{abstract}

Keywords: linear-quadratic regulator, Anti-Tank Guided Missile, direction algorithm, Jacobian

\section{Introduction}

The linear-quadratic regulator is best suited for controlling automation objects, which can be subjected to linearization and their point of work is fixed. In the case when dynamic equations are strongly non-linear and non-stationary, their linearization causes significant errors or is often simply impossible. The ATGM is a control object the parameters of which change in time. This applies mostly to mass, moments of inertia, centre of mass location, aerodynamic coefficients, velocity of flight, engine thrust, etc. The position angles of the velocity vector and their derivatives with respect to time change during the flight depending on the implemented control algorithm.

Control of manoeuvring the ATGM using variable engine thrust geometry is more and more common. There are hybrid control systems consisting of at least two executive mechanisms, wherein one is vectorization of thrust and the other are aerodynamic surfaces. They can be supported with internal gasodynamic controls which are activated within the initial stage of flight when the missile accelerates and the forces generated on the control surfaces are insufficient. One of the ATGMs which employs control assistance using added control surfaces located at the outlet of the engine is "Javelin" (Fig. 1). This causes a change of the engine thrust direction.

In the paper (Harris and Slegers, 2009), the authors investigated the impact of a defect of control surfaces and stabilizers on the control capabilities. Another example of the issue concerning the control and stability of the ATGM as a non-stationary system is the paper by Guo et al. (2017). Based on the analysis of the available bibliography, it seems that the ATGM guiding algorithm using Jacobians (called the modified LQR method in this paper) has never been used. 


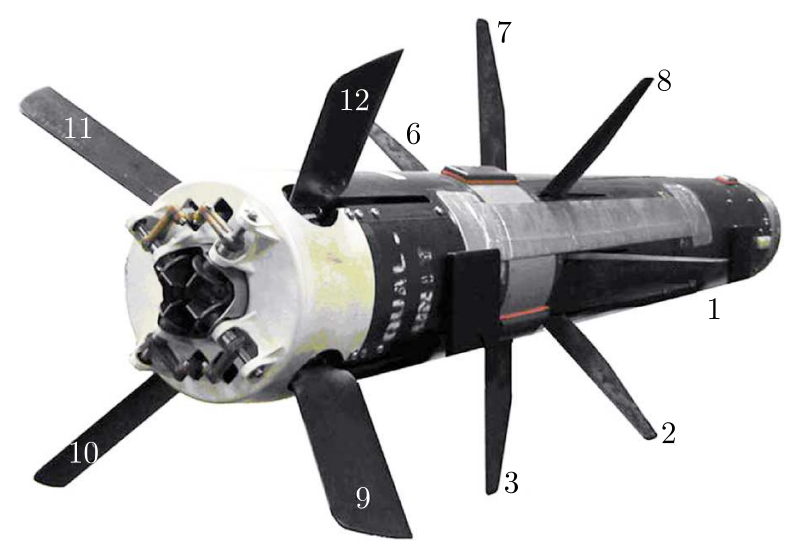

Fig. 1. General view of "Javelin" - the anti-tank guided missile of the latest generation (Harris and Slegers, 2009)

\section{Controlled object}

The controlled object is a subsonic, hypothetical short or medium range anti-tank guided missile, i.e. "fire and forget" (Fig. 2a). It is controlled using a double executive control system. The executive system consists of two pairs of aerodynamic controls in the front part of the missile and a tilting nozzle of the engine (Fig. 2b), which changes the angular position of the thrust vector in relation to the longitudinal axis of the missile $S x$. The application of the hybrid executive system significantly improves manoeuvrability of the missile, as presented in the paper (Nocon and Koruba, 2017).
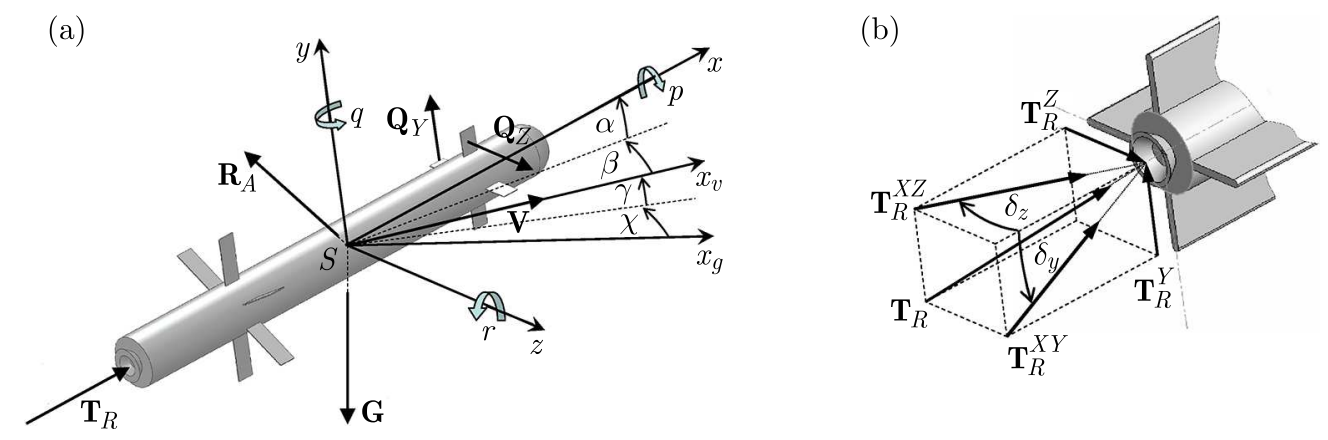

Fig. 2. (a) Force system acting on the ATGM within the gravitational field and Earth atmosphere together with the accepted coordinate systems. (b) Generation of control forces while tilting the engine nozzle

Figure 2 uses the following symbols and designations: $\mathbf{R}_{A}$ - vector of resultant aerodynamic forces; $\mathbf{T}_{R}$ - engine thrust; $\mathbf{G}$ - force of gravity; $\mathbf{Q}_{Y}, \mathbf{Q}_{Z}$ - control forces; $\mathbf{V}$ - vector of missile velocity; $S x_{g} y_{g} z_{g}$ - ground-fixed coordinate system; $S x_{v} y_{v} z_{v}$ - coordinate system connected with the flow; Sxyz - coordinate system connected with the missile; $\alpha$ - angle of attack; $\beta$ missile sideslip angle; $\gamma$ and $\chi$ - angles of the velocity vector (flight-path angles); $p, q, r$ - angular velocity components in the body-fixed system.

Deviation of the engine thrust vector from the longitudinal axis of the ATGM by the angles $\delta_{y}, \delta_{z}$ (Fig. 2b) provides control forces in both planes. In the vertical plane, the flight height control force is generated $\mathbf{T}_{R}^{Y}$, in the horizontal plane, the flight direction control force is generated $\mathbf{T}_{R}^{Z}$ (Koruba and Nocoń, 2016b).

It is assumed that $\mathbf{T}_{R}^{Y}$ is the thrust projection on the vertical axis $S y ; \mathbf{T}_{R}^{Z}$ - thrust projection on the horizontal axis $S z ; \mathbf{T}_{R}^{X}$ - thrust projection on the longitudinal axis of the ATGM $S x$. 
The following thrust vector deviation angles are adopted: $\delta_{z}$ - control angle in the horizontal plane; $\delta_{y}$ - control angle in the vertical plane.

The trigonometry shows that: $T_{R}^{X}=T_{R}^{X Z} \cos \delta_{z}, T_{R}^{Z}=T_{R}^{X Z} \sin \delta_{z}$ - in the horizontal plane $S x z ; T_{R}^{X}=T_{R}^{X Y} \cos \delta_{y}, T_{R}^{Y}=T_{R}^{X Y} \sin \delta_{y}$ - in the vertical plane $S x y$. The control forces $\mathbf{T}_{R}^{Y}$ and $\mathbf{T}_{R}^{Z}$ are derived in detail in the article (Koruba and Nocon, 2016b), they are equal to

$$
\begin{aligned}
& T_{R}^{Y}=\left|\mathbf{T}_{R}^{Y}\right|=\operatorname{sgn}\left(\delta_{y}\right) \sqrt{\frac{T_{R}^{2} \cos ^{2} \delta_{z} \sin ^{2} \delta_{y}}{1-\sin ^{2} \delta_{z} \sin ^{2} \delta_{y}}} \approx T_{R} \delta_{y} \\
& T_{R}^{Z}=\left|\mathbf{T}_{R}^{Z}\right|=\operatorname{sgn}\left(\delta_{z}\right) \sqrt{\frac{T_{R}^{2} \cos ^{2} \delta_{y} \sin ^{2} \delta_{z}}{1-\sin ^{2} \delta_{z} \sin ^{2} \delta_{y}}} \approx T_{R} \delta_{z}
\end{aligned}
$$

Aerodynamic Controls (AC) are located at the missile front. The light composite construction of AC (Chatys, 2013) provides a quick response to control signals. In the case of small deflections of $\mathrm{AC} \alpha_{Y}, \alpha_{Z}$ (Nocon, 2017) carrying and side (control) forces $Q_{Y}, Q_{Z}$ generated on the AC, adopt simplified form (2.2). The pair of AC in the front part of the missile can be described using the following formulas

$$
Q_{Y}=2 \alpha_{Y} S_{S} \rho \frac{V^{2}}{2} \quad Q_{Z}=2 \alpha_{Z} S_{S} \rho \frac{V^{2}}{2}
$$

where: $S_{S}$ - area of surfaces $\left(S_{S}=0.009 \mathrm{~m}^{2}\right), \rho$ - air density $\left(\rho=1.225 \mathrm{~kg} / \mathrm{m}^{3}\right)$.

The controlled object is guided to a target using an optical scanning seeker (Gapiński et al., 2014) or, in the case of medium range, using a television head which enables operation such as "fire, observe, correct". It is assumed that the guided missile operates correctly and the data on missile location versus its target is known. Moreover, it is assumed that the AGTM does not make any rotational motion in relation to axis $S x$. Simplifying assumptions are also made, which slightly affect the errors in simulation calculations. Initial studies are performed on a physical model in the vertical plane (Fig. 2), and it results that the angle of attack is $\alpha=\Theta-\gamma, \Theta$ - pitch angle.

It is assumed that the ATGM mass changes due to consumption of the engine fuel. Thus, the center of mass moves and moments of inertia change. These changes are described using time-dependent linear functions.

Calculations shown in Fig. 3 are made for: $m_{0}$ - initial mass of the ATGM is $13.287 \mathrm{~kg}$, $m_{1}$ - mass of the ATGM after consumption of fuel is $8.7 \mathrm{~kg}, x_{s m 0}$ - initial position of the center of mass of the ATGM $=0.5705 \mathrm{~m}$ (when viewed from the front of the ATGM), $x_{s m 1}$ - position of the center of mass of the ATGM after consumption of fuel is $0.434 \mathrm{~m}$ (when viewed from the front of the ATGM), $I_{x 0}$ - initial moment of inertia of the ATGM in relation to $x$ axis is $0.0396 \mathrm{kgm}^{2}, I_{x 1}-$ moment of inertia of the ATGM in relation to $x$ axis after consumption of the fuel is $0.026 \mathrm{kgm}^{2}, I_{y 0}=I_{z 0}$ - initial moment of inertia of the ATGM in relation to the transverse axis is $1.7823 \mathrm{kgm}^{2}, I_{y 1}=I_{z 1}$ - moment of inertia of the ATGM in relation to the transverse axis after consumption of the fuel is $1.2078 \mathrm{kgm}^{2}$.

Full dynamic equations of spatial flight of any flying object as a rigid solid body can be found, among others, in the papers (Koruba and Nocoń, 2016a; Nocoń and Stefański, 2016; Baranowski 2013). For the purpose of the analysis of the modified LQR method algorithm, dynamic equations of the ATGM considered herein have been simplified to the vertical plane, making the following assumptions

$$
p=0 \quad q=0 \quad r=\dot{\Theta}
$$


(a)

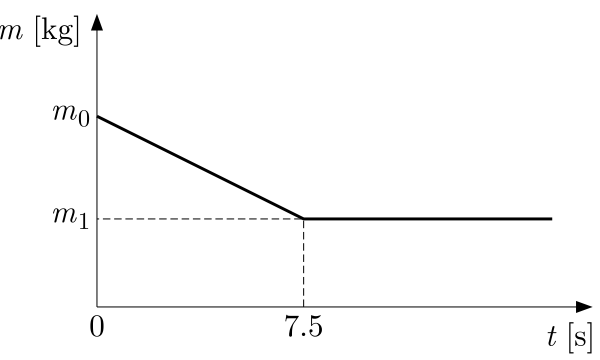

(c)

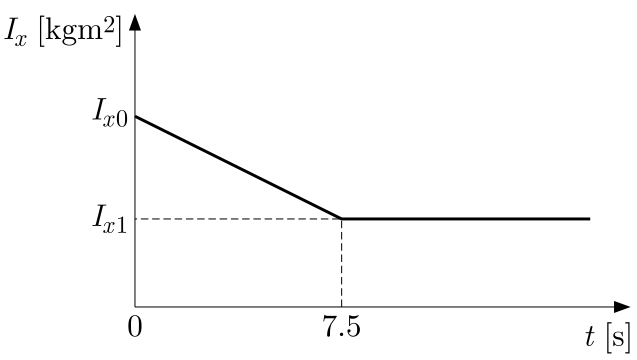

(b)

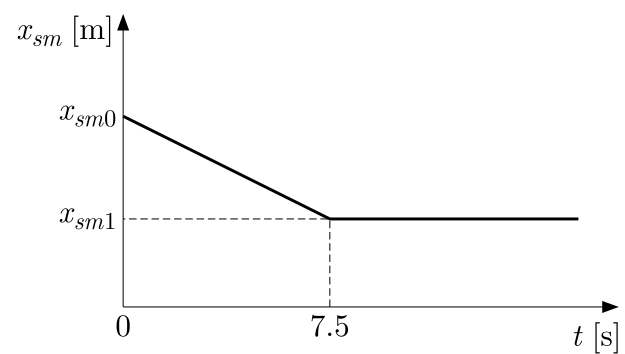

(d)

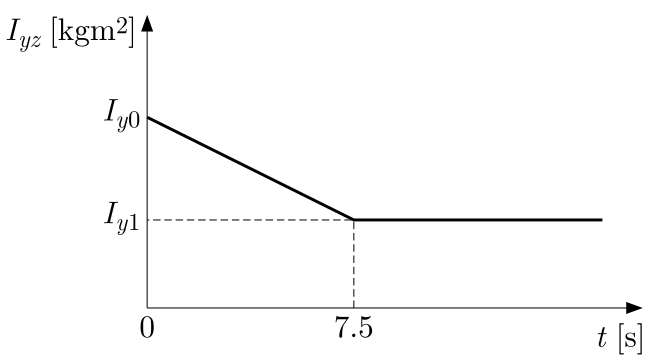

Fig. 3. (a) Mass change, (b) center of mass change, (c) moments of inertia change, (d) moments of inertia change

Then, these equations have the following form

$$
\begin{aligned}
& m \dot{V}=T_{R} \cos \alpha-G \sin \gamma-m \lambda_{x} V^{2} \\
& m V \dot{\gamma}=T_{R} \sin \alpha-G \cos \gamma+m \lambda_{y} V^{2} \alpha+Q_{Y}+T_{R}^{Y} \\
& \ddot{\Theta}=-D_{1} \frac{\alpha}{L_{P}} V^{2}-D_{2} V \dot{\alpha}-D_{3} V \dot{\Theta}+e \frac{Q_{Y}}{I_{z}}+f \frac{T_{R}^{Y}}{I_{z}}
\end{aligned}
$$

where: $T_{R}$ - engine thrust, $e=x_{S m}-x_{S}, f=x_{S m}-L_{P}$ - distance from the center of mass of $\mathrm{AC}$ and outlet gas nozzles, correspondingly, $x_{s}$ - coordinates of AC center (when viewed from the front of the ATGM), $L_{P}$ - length of the ATGM body, $I_{z}$ - moments of inertia in relation to the axes of the related system, $m=m(t)$ - mass of the missile, $\Theta$ - pitch angle, $\lambda_{X}, \lambda_{Y}$, $D_{i}=C_{i} L_{P} / I_{z}$ - relative aerodynamic factors of forces and moment, $C_{1}-$ lift and drift moment factors, $C_{2}, C_{3}$ - damping moment factors from the ATGM body angular velocities (constans values were adopted) (Koruba and Osiecki, 2006).

It is assumed that the deflection angle of the AC and engine nozzle have the same values but opposite directions. In the vertical plane, we have $\alpha_{Y}=-\delta_{y}$ which results in the following equations

$$
Q_{Y}=\alpha_{Y} P_{S} \quad T_{R}^{Y} \approx-T_{R} \alpha_{y}
$$

where: $P_{S}=2 S_{S} \rho V^{2} / 2$.

\section{The control algorithm in the modified LQR method}

The general idea of the programmed trajectory is derived from the paper (Koruba and Nocon, 2016a). Determining the programmed (set) trajectory consists in finding a polynomial function of the third degree that binds the initial and final point of the flight trajectory section. These points are called flight points. There can be a few such trajectory sections. The last section connects the ATGM with a target. The suitable control angles are selected so that the ATGM 
flight could correspond to the programmed trajectory - this is an essential control task. The modified linear-quadratic regulator is used for this purpose.

The dynamic equations of the AGTM flight in the vertical plane are strongly non-linear, and their linearization is practically impossible due to significant errors resulting from major simplifications. In the paper (Koruba and Nocon, 2016b), it was proven that a linear-quadratic regulator may be used to regulate this type of objects, however with time-fixed states matrices $\mathbf{A}$ and control matrices $\mathbf{B}$, the mathematical model is far from the current one. The modified LQR method solves this problem. It determines the control angle $\alpha_{Y}=-\delta_{y}$ for non-linearized flight dynamics equations (2.4). The control signals determined by this method are the angle of deflection of the $\mathrm{AC}$ and the nozzle of the engine.

Within the determined state, at a given point of work, the dynamic equations assume the form of a linear function. In the case considered by us, the ATGM point of work is any consecutive point of the missile flight position during guidance to the target (implementation of the firing task). The linearization of the dynamic equations is performed at any time instant - around the next point of work.

For the purpose of developing the control with the modified LQR method, dynamic equations (2.4) have been narrowed down to the equations of state

$$
\begin{aligned}
& \frac{d V}{d t}=\frac{T_{R}}{m} \cos (\Theta-\gamma)-g \sin \gamma-\lambda_{x} V^{2} \\
& \frac{d \gamma}{d t}=\frac{T_{R}}{m V} \sin (\Theta-\gamma)-\frac{g}{V} \cos \gamma+\lambda_{y}(\Theta-\gamma) V+\frac{Q_{Y}}{m V}+\frac{T_{R}^{Y}}{m V} \\
& \frac{d \dot{\Theta}}{d t}=\ddot{\Theta} \\
& \ddot{\Theta}=-D_{1} \frac{\Theta-\gamma}{L_{P}} V^{2}-D_{2} V(\dot{\Theta}-\dot{\gamma})-D_{3} V \dot{\Theta}+e \frac{Q_{Y}}{I_{Z}}+f \frac{T_{R}^{Y}}{I_{Z}}
\end{aligned}
$$

Assuming that the system of dynamic equations (3.1) is linearized at any time instant, it can have the form of a vector-matrix equation

$$
\Delta \dot{\mathbf{x}}(t)=\mathbf{J}(t) \Delta \mathbf{x}(t)+\mathbf{B u}
$$

where: $\dot{\mathbf{x}}(t)=[\dot{V}, \dot{\gamma}, \dot{\Theta}, \ddot{\Theta}]^{\mathrm{T}} ; \Delta \mathbf{x}(t)=\mathbf{x}(t)-\mathbf{x}_{Z}(t)$ - vector of state variables deviations from the required value; $\mathbf{x}(t)=[V, \gamma, \Theta, \dot{\Theta}]^{\mathrm{T}}$ - vector of actual state variables; $\mathbf{x}_{Z}(t)$ - vector of state variables at the point of work, i.e. vector of required values of state variables; $\mathbf{u}(t)=\left[\alpha_{Y}\right]-$ vector of forcing (control of) the system; $\mathbf{J}$ - Jacobian, system state matrix, calculated within each sample time; $\mathbf{B}$ - control matrix.

It must be emphasized that calculation of Jacobian at each time instant allows one to shift the point of work to a place where the ATGM is currently present. The data necessary for adjustment using the LQR method derives directly from the mathematical model given in the form of dynamic equations described with formulas (2.4). At each time instant for the LQR, a new Jacobian $\mathbf{J}$ and control matrix $\mathbf{B}$ is calculated, so is the matrix of amplification $\mathbf{K}$.

The Jacobian of the system described with equations (3.1) is presented in the following form

$$
\mathbf{J}=\left[\begin{array}{llll}
\frac{\partial \dot{V}}{\partial V} & \frac{\partial \dot{V}}{\partial \gamma} & \frac{\partial \dot{V}}{\partial \Theta} & \frac{\partial \dot{V}}{\partial \dot{\Theta}} \\
\frac{\partial \dot{\gamma}}{\partial V} & \frac{\partial \dot{\gamma}}{\partial \gamma} & \frac{\partial \dot{\gamma}}{\partial \Theta} & \frac{\partial \dot{\gamma}}{\partial \dot{\Theta}} \\
\frac{\partial \dot{\Theta}}{\partial V} & \frac{\partial \dot{\Theta}}{\partial \gamma} & \frac{\partial \dot{\Theta}}{\partial \Theta} & \frac{\partial \dot{\Theta}}{\partial \dot{\Theta}} \\
\frac{\partial \ddot{\Theta}}{\partial V} & \frac{\partial \ddot{\Theta}}{\partial \gamma} & \frac{\partial \ddot{\Theta}}{\partial \Theta} & \frac{\partial \ddot{\Theta}}{\partial \dot{\Theta}}
\end{array}\right]_{x_{0}}
$$


The matrix $\mathbf{J}$ is a Jacobian of simultaneous equations (3.1) and consists of partial derivatives calculated at each point of work $x_{0}\left(V_{0}, \gamma_{0}, \Theta_{0}, \dot{\Theta}_{0}\right)$.

The mentioned partial derivatives have the following forms

$$
\begin{aligned}
& \frac{d \dot{V}}{d V}=-2 \lambda_{x} V_{0} \quad \frac{d \dot{V}}{d \gamma}=\frac{T_{R}}{m} \sin \left(\Theta_{0}-\gamma_{0}\right)-g \cos \gamma_{0} \\
& \frac{d \dot{V}}{d \Theta}=-\frac{T_{R}}{m} \sin \left(\Theta_{0}-\gamma_{0}\right) \quad \frac{d \dot{V}}{d \dot{\Theta}}=0 \\
& \frac{d \dot{\gamma}}{d V}=-\left(\frac{T_{R}}{m} \sin \left(\Theta_{0}-\gamma_{0}\right)-g \cos \gamma_{0}\right) \frac{1}{V_{0}^{2}}+\lambda_{y}\left(\Theta_{0}-\gamma_{0}\right) \quad \frac{d \dot{\gamma}}{d \dot{\Theta}}=0 \\
& \frac{d \dot{\gamma}}{d \gamma}=-\frac{T_{R}}{m V_{0}} \cos \left(\Theta_{0}-\gamma_{0}\right)+\frac{g}{V_{0}} \sin \gamma_{0}-\lambda_{y} V_{0} \quad \frac{d \dot{\gamma}}{d \Theta}=\frac{T_{R}}{m V_{0}} \cos \left(\Theta_{0}-\gamma_{0}\right)-\lambda_{y} V_{0} \\
& \frac{d \dot{\Theta}}{d V}=0 \quad \frac{d \dot{\Theta}}{d \Theta}=0 \quad \frac{d \dot{\Theta}}{d \dot{\Theta}}=0 \quad 1 \\
& \frac{d \ddot{\Theta}}{d V}=-2 \frac{D_{1}}{L_{P}}\left(\Theta_{0}-\gamma_{0}\right) V_{0}-D_{2} \dot{\Theta}_{0}+2 D_{2} V_{0} \lambda_{y}\left(\Theta_{0}-\gamma_{0}\right)-D_{3} \dot{\Theta}_{0} \\
& \frac{d \ddot{\Theta}}{d \gamma}=\frac{D_{1}}{L_{P}} V_{0}^{2}-D_{2} \frac{T_{R}}{m} \cos \left(\Theta_{0}-\gamma_{0}\right)+D_{2} g \sin \gamma_{0}-D_{2} V_{0}^{2} \lambda_{y} \\
& \frac{d \ddot{\Theta}}{d \Theta}=-\frac{D_{1}}{L_{P}} V_{0}^{2}+D_{2} \frac{T_{R}}{m} \cos \left(\Theta_{0}-\gamma_{0}\right)+D_{2} V_{0}^{2} \lambda_{y} \quad \frac{d \ddot{\Theta}}{d \dot{\Theta}}=-D_{2} V_{0}-D_{3} V_{0}
\end{aligned}
$$

However, the control matrix $\mathbf{B}$ has the following form

$$
\mathbf{B}=\left[\begin{array}{c}
0 \\
\frac{P_{S}}{m V}-\frac{T_{R}}{m V} \\
0 \begin{array}{c}
D_{2} P_{S} \\
m
\end{array}+e \frac{P_{S}}{J_{z}}-\frac{D_{2} T_{R}}{m}-f \frac{T_{R}}{J_{z}}
\end{array}\right]
$$

The right control $\mathbf{u}=\mathbf{u}(t)$ has been determined, which minimizes the square index of quality

$$
J=\frac{1}{2} \int_{0}^{t k} \mathbf{x}^{\mathrm{T}}(t) \mathbf{Q} \mathbf{x}(t)+\mathbf{u}^{\mathrm{T}}(t) \mathbf{R u}(t) d t
$$

where: $\mathbf{Q}$ - symmetrical matrix positively semi-definite; $\mathbf{R}$ - symmetrical matrix positively definite.

Based on the dynamic equations, the gain matrix of the LQR has been determined

$$
\mathbf{K}=\mathbf{R}^{-1} \mathbf{B}^{\mathrm{T}} \mathbf{S}
$$

each of them allows for minimization of square quality index (3.5). The minimization process includes solution of the algebraic Riccati equation which can be determined using the function in Matlab (Tewari, 2002).

The matrices $\mathbf{Q}, \mathbf{R}$ are selected for two time intervals. The first time interval is the time when the launch motor is on $(0 \mathrm{~s} \leqslant t<0.5 \mathrm{~s})$ and the thrust is $4.3 \mathrm{kN}$. The second interval is when the main engine is on $(0.5 \mathrm{~s} \leqslant t<7.5 \mathrm{~s})$ the thrust of which is $280 \mathrm{~N}$ 


$$
\begin{aligned}
& \mathbf{Q}_{t<0.5 \mathrm{~s}}=\left[\begin{array}{cccc}
0.474 & 0 & 0 & 0 \\
0 & 1.6 & 0 & 0 \\
0 & 0 & 70.6 & 0 \\
0 & 0 & 0 & 0.00521
\end{array}\right] \quad \mathbf{R}_{t<0.5 \mathrm{~s}}=[0.082] \\
& \mathbf{Q}_{0.5 \mathrm{~s} \leqslant t}=\left[\begin{array}{cccc}
0.001 & 0 & 0 & 0 \\
0 & 0.01 & 0 & 0 \\
0 & 0 & 0.01 & 0 \\
0 & 0 & 0 & 0.151
\end{array}\right] \quad \mathbf{R}_{0.5 \mathrm{~s} \leqslant t}=[0.0391]
\end{aligned}
$$

It is assumed that the linear system is in balance. The purpose of the control is to maintain it within a set point of work, despite the interruptions affecting it. In this case, the AGTM moves and the point of work is not fixed. The balance state depends on the deviation $\mathbf{x}(t)-\mathbf{x}_{Z}(t)$. The optimum control can have the following form

$$
\mathbf{u}(t)=-\mathbf{K}\left[\mathbf{x}(t)-\mathbf{x}_{Z}(t)\right]
$$

When substituting equation (3.8) to (3.2), one gets

$$
\Delta \dot{\mathbf{x}}(t)=(\mathbf{J}-\mathbf{B K})\left[\mathbf{x}(t)-\mathbf{x}_{Z}(t)\right]
$$

The vector of the set state variables $\mathbf{x}_{Z}(t)=\left[V_{0}, \gamma^{o}+\gamma_{Z}, \Theta_{0}, \dot{\Theta}_{0}\right]^{\mathrm{T}}$ is derived from data calculated based on the programmed trajectory.

The programmed flight trajectory in the vertical plane is a polynomial of the third degree $y_{Z}$, based on which the set flight height control angle $\gamma_{Z}$ is calculated

$$
\gamma_{Z}=\arctan \left(3 a_{y} x^{2}+2 b_{y} x+c_{y}\right)
$$

Moreover, height correction in the form of an angle is introduced into the vector of set variables. Angular correction of the position results from trigonometry. The form is as follows

$$
\tan \gamma^{o}=\frac{\Delta y \cos \gamma_{Z}}{\Delta x} \Longrightarrow \gamma^{o}=\arctan \left(\frac{\Delta y}{\Delta x} \cos \gamma_{Z}\right)
$$

where: $a_{y}, b_{y}, c_{y}$ - polynomial coefficients calculated according to the control algorithm (Koruba and Nocon, 2016a); $\Delta y=y_{Z}-y ; y_{Z}$ - programmed trajectory coordinates; $y$ - real ATGM coordinates; $\Delta x$ - projected distance necessary to correct the ATGM flight - assumed as $\Delta x=10 \mathrm{~m}$.

\section{Results of computer simulation}

The simulation has been performed for a hypothetical missile, equation (2.4), controlled using a modified LQR. The executive system of the control is a double system consisting of aerodynamic surfaces in the front part of the missile and variable geometry of engine thrust. Geometrical and mass properties of the ATGM and the remaining parameters were selected as follows: $L_{P}=1.2 \mathrm{~m}, x_{s}=0.2 \mathrm{~m}, D_{1}=0.081 \mathrm{~m}^{-1}, D_{2}=0.0821 \mathrm{~m}^{-1}, D_{3}=0.00041 \mathrm{~m}^{-1}$, $\lambda_{x}=0.000171 \mathrm{~m}^{-1}, \lambda_{y}=0.051 \mathrm{~m}^{-1}$.

The simulation has been performed for the following initial conditions.

The point of start of the ATGM is located at the beginning of the coordinate system $O x_{g} y_{g} z_{g}$, initial velocity of the ATGM $V_{0}=50 \mathrm{~m} / \mathrm{s}$, sample time $d t=0.001 \mathrm{~s}$, pitch angle of starting $\Theta=0$.

Simulation of guiding the ATGM to a target has been carried out at velocity $V_{T}=30 \mathrm{~m} / \mathrm{s}$ and angle of target velocity vector $\gamma_{T}=10^{\circ}$, spaced from the shooting position by $750 \mathrm{~m}$, located at a height of $2 \mathrm{~m}$ in relation to the missile. The flight point of the ATGM has the coordinates $P_{1}(400 ; 10)$.

The simulation has been performed for the target moving at velocity $V_{T}=30 \mathrm{~m} / \mathrm{s}$ and angle $\gamma_{T}=10^{\circ}$, spaced from the shooting position by $2300 \mathrm{~m}$, located at a height of $1 \mathrm{~m}$. The points of flight of the ATGM are $P_{1}(400 ; 10), P_{2}(750 ; 2), P_{3}(1200 ; 20)$. 


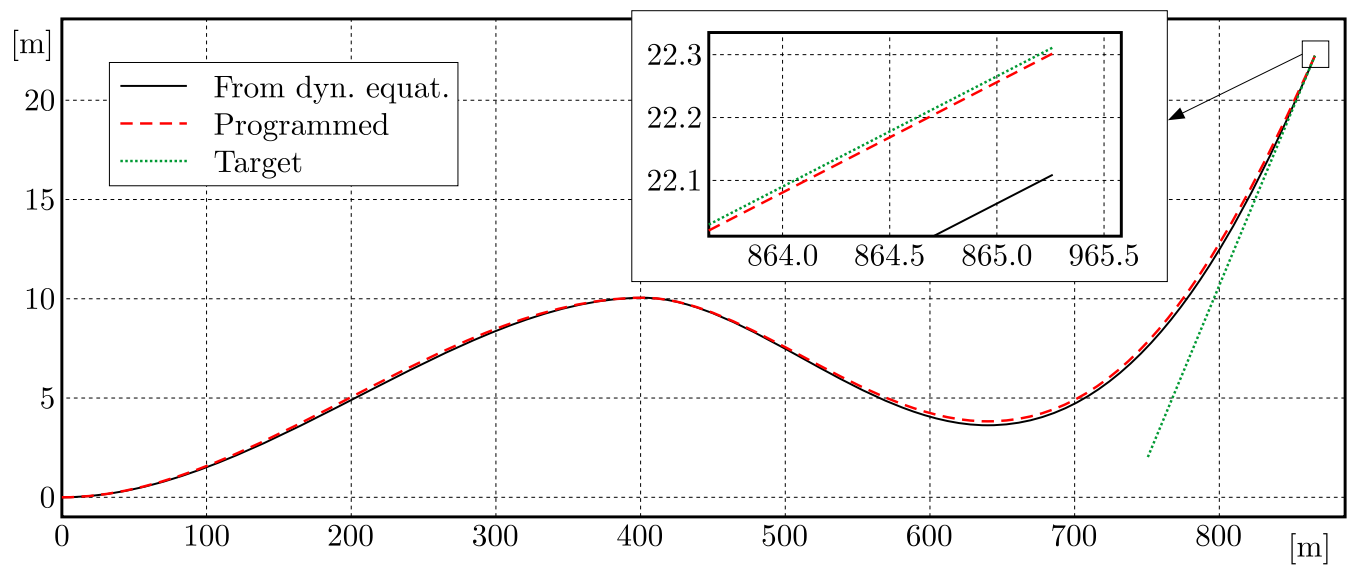

Fig. 4. Set and implemented ATGM flight path and the path to a target in a vertical plane. The implemented flight of the ATGM is calculated based on the flight dynamic equations, however, the set trajectory results from the control algorithm. The point of flight is $P_{1}(400 ; 10)$

(a)

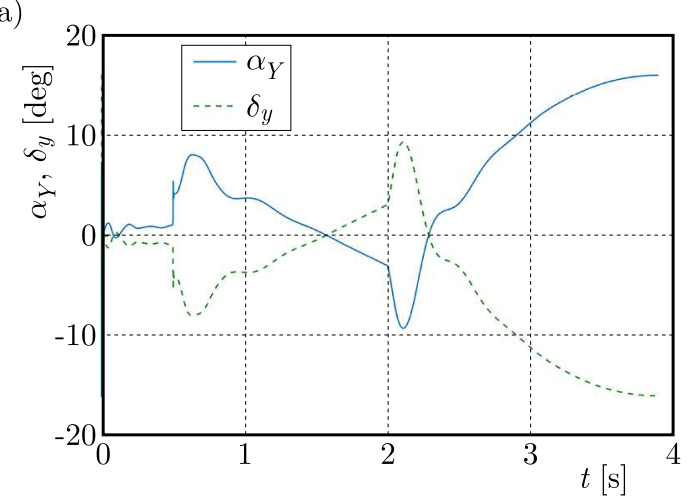

(b)

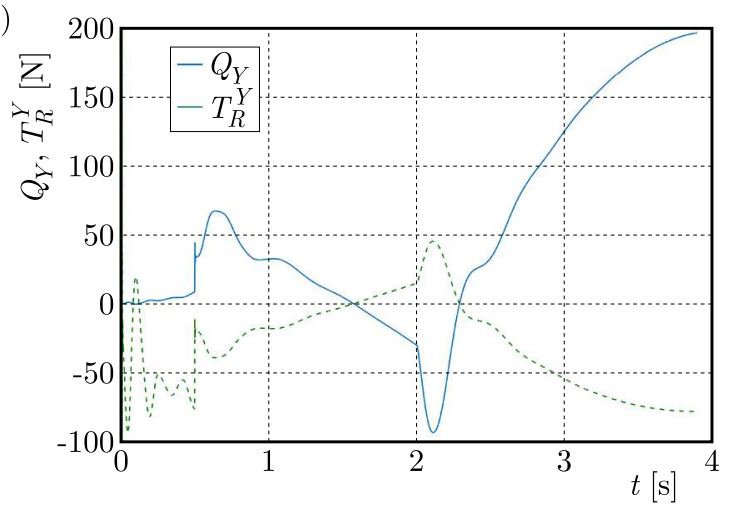

Fig. 5. (a) Implemented deflection angles of aerodynamic surfaces $\alpha_{Y}$ and nozzle deflection $\delta_{y}$, for the height control channel. (b) Flight height control forces generated on aerodynamic surfaces $Q_{Y}$ and using the engine nozzle $T_{R}^{Y}$ in function of time

(a)

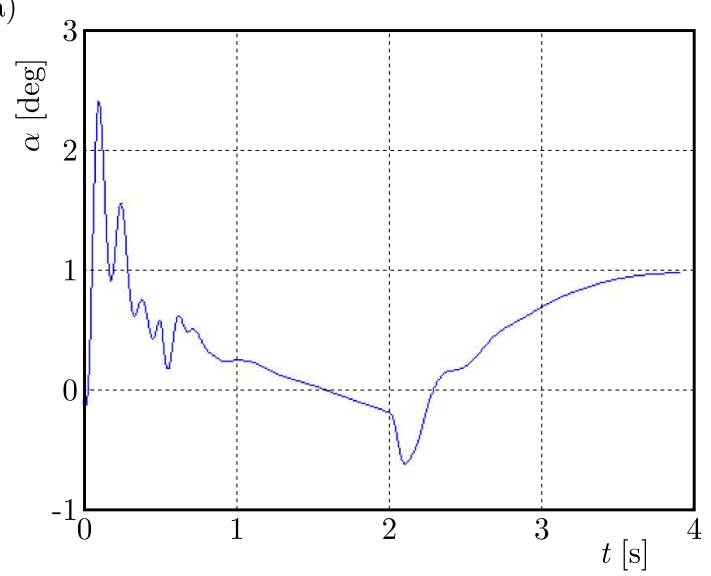

(b)

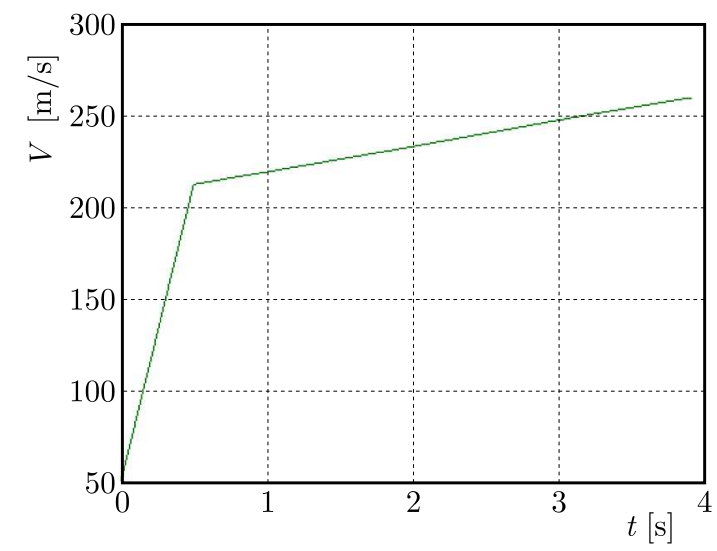

Fig. 6. Implemented angle of attack $\alpha$ (a) and velocity of the ATGM (b) in function of time

\section{Final conclusions, direction of further studies}

The initial studies have been performed on a mathematical model of the ATGM motion in a vertical plane. The algorithm of a modified LQR method using Jacobian for the ATGM control 


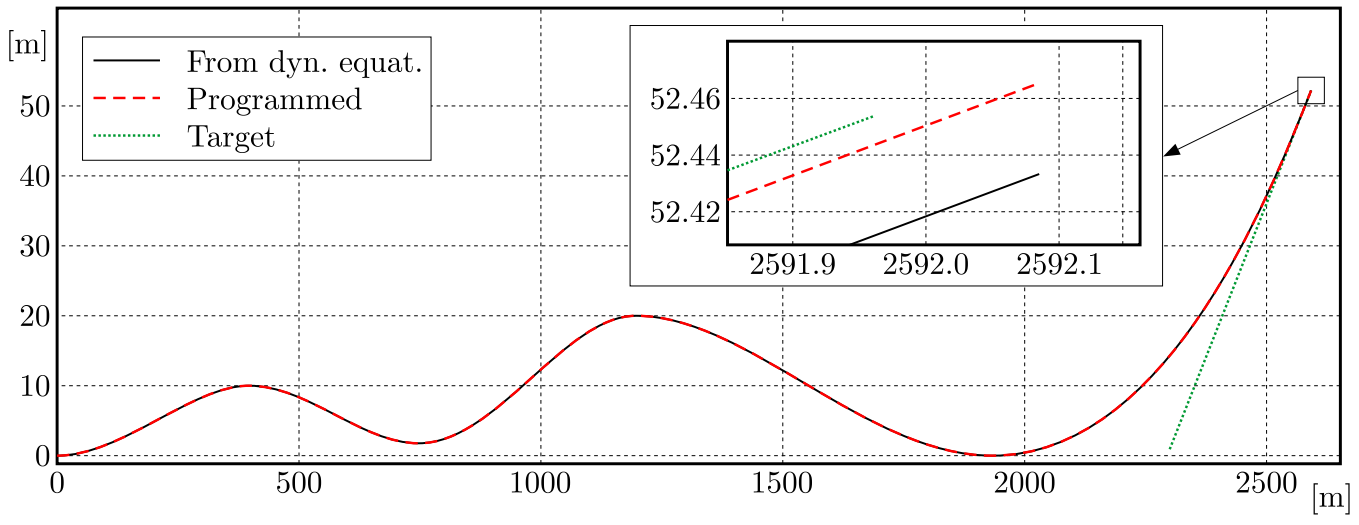

Fig. 7. Set and implemented ATGM flight path and the path to a target in a vertical plane. The implemented flight of the ATGM is calculated based on the flight dynamic equations, however, the set trajectory results from the control algorithm. The points of flight are $P_{1}(400 ; 10)$, $P_{2}(750 ; 2), P_{3}(1200 ; 20)$

(a)

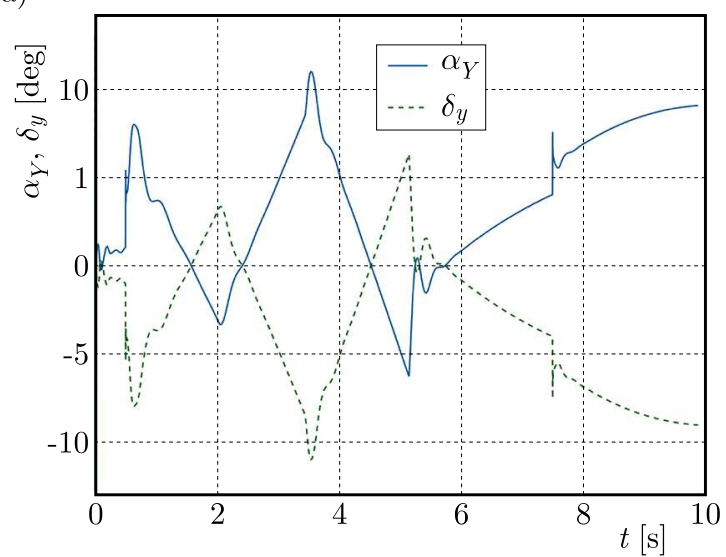

(b)

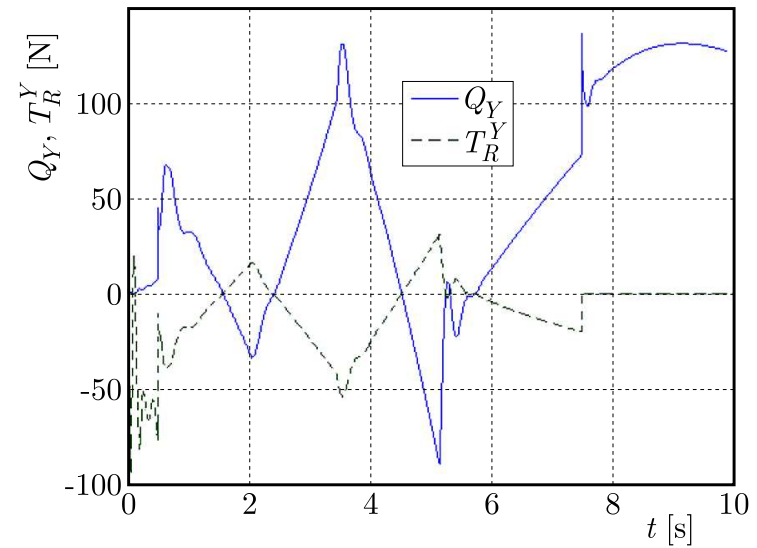

Fig. 8. (a) Implemented deflection angles of aerodynamic surfaces $\alpha_{Y}$ and nozzle deflection $\delta_{y}$, for the height control channel. (b) Flight height control forces generated on aerodynamic surfaces $Q_{Y}$ and using the engine nozzle $T_{R}^{Y}$ in function of time

(a)

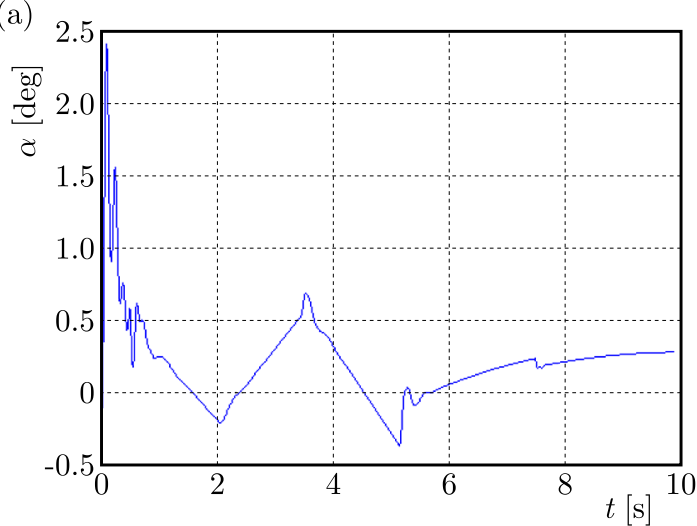

(b)

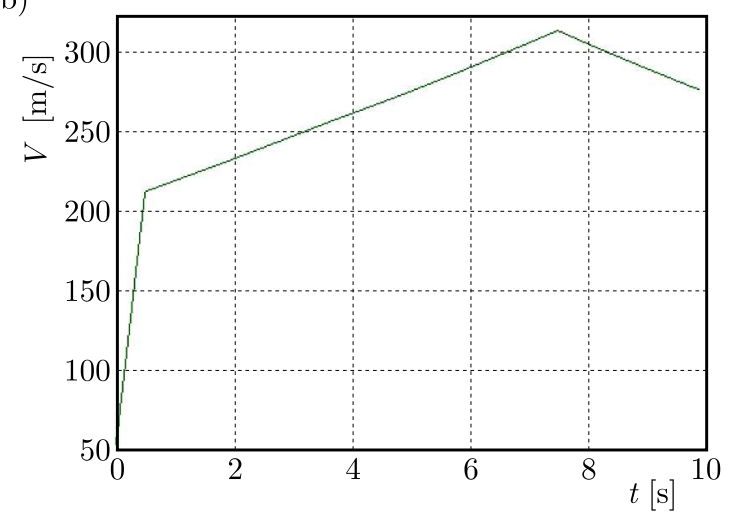

Fig. 9. Implemented angle of attack $\alpha$ (a) and velocity of the missile (b) in function of time 
presented in the paper works correctly. The missile mirrors the set trajectory with deviation not exceeding $0.4 \mathrm{~m}$. The target is hit with an accuracy of $0.2 \mathrm{~m}$. The modified regulator develops control signals (deviation angles of the aerodynamic surface and the vector of engine thrust) based on dynamic equations. It must be underlined that the algorithm considers limitations related to the aforementioned controls - values of deviation angles do not exceed 15 degrees.

Application of time-variable elements of the matrix $\mathbf{J}(t), \mathbf{B}(t)$ allowed for linearization of the flight dynamic equations within a subsequent sample time. The dynamic character of flight equations has been thus maintained. In practice, Jacobian is applied as a matrix $\mathbf{J}$ - matrix consisting of partial derivatives. It results in an option to control a strongly non-linear and non-stationary object using LQR.

Satisfactory results of the application of the modified LQR for the control of an anti-tank guided missile in a vertical plane encourage further studies of the full spatial model. Moreover, in the case of lack of access to all state variables, it will be necessary to use a proper state observer, and in the case of random interferences - Kalman filter.

\section{References}

1. BarAnowski L., 2013, Effect of the mathematical model and integration step on the accuracy of the results of computation of artillery projectile flight parameters, Bulletin of the Polish Academy of Sciences - Technical Sciences, 61, 2, 475-484

2. Chatys R., 2013, Investigation of the effect of distribution of the static strength on the fatigue failure of a layered composite by using the Markov chains theory, Mechanics of Composite Materials, 48, 6, 629-638

3. Gapinski D., Koruba Z., Krzysztofik I., 2014, The model of dynamics and control of modified optical scanning seeker in anti-aircraft rocket missile, Mechanical Systems and Signal Processing, 45, 2, 433-447

4. Guo X., Li Z., Zhai Y., Liu D., 2017, Design on a lag-lead emendation network for some missile control system, EURASIP Journal on Wireless Communications and Networking, 2017, 12

5. Harris J., Slegers N., 2009, Performance of a fire-and-forget anti-tank missile with a damaged wing, Mathematical and Computer Modelling, 50, 1-2, 292-305

6. Koruba Z., Nocoń Ł., 2016a, Numerical analysis of the dynamics of automatically tracked antitank guided missile using polynomial functions, Journal of Theoretical and Applied Mechanics, 54, $1,13-25$

7. Koruba Z., Nocoń Ł., 2016b, Optimal compensator for anti-ship missile with vectorization of engine thrust, Applied Mechanics and Materials, 817, 279-288

8. Koruba Z., Osiecki J., 2006, Structure, Dynamics and Navigation of Chosen Precision Kill Weapons, Publishing House of Kielce University of Technology, ISBN 83-88906-17-8, Kielce

9. NoCoń Ł., 2017, Development and analysis of anti-tank guiding algorithms of the third generation missile with the possibility of bypassing obstacles, PhD Thesis, Kielce University of Technology, Kielce

10. Nocoń Ł., Koruba Z., 2017, Modifications of control actuation systems of ATGM, 23rd International Conference on Engineering Mechanics 2017, Svratka, Czech Republic, 714-717

11. Nocoń Ł., Stefański K., 2016, Impact of controller performance on the process of guiding an armour-piercing missile onto a ground-based target, Problemy Mechatroniki: Uzbrojenie, Lotnictwo, Inżynieria Bezpieczeństwa, 7, 4(26), 67-84

12. Tewari A., 2002, Modern Control Design with Matlab and Simulink, Jon Wiley \& Sons, LTD 